\title{
A Report from the NIMBioS/DySoC Investigative Workshop on the Mathematics of Gun Violence
}

\author{
Shelby Scott
}

January 2, 2020 


\section{Contents}

1 Introduction . . . . . . . . . . . . . . . . . . . . . . . . . . . . . . . . . . . . . . . . . . . . 2

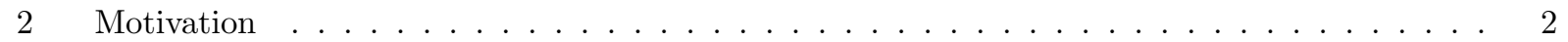

3 Collaboration with Stakeholders . . . . . . . . . . . . . . . . . . . . . . . . . . . . . . 5

4 Intervention . . . . . . . . . . . . . . . . . . . . . . . . . . . . . . . . . . . . 6

5 Quantifying the Impact of Rare Event Interventions . . . . . . . . . . . . . . . . . . . . . . . 8

6 Epidemiological Criminology . . . . . . . . . . . . . . . . . . . . . . . . . . . . . . . . . . 9

7 Theoretical Models . . . . . . . . . . . . . . . . . . . . . . . . . . . . . . 10

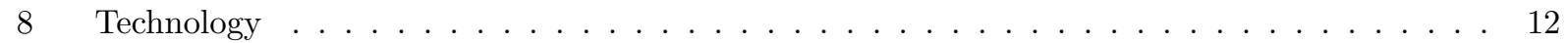

9 Data Use . . . . . . . . . . . . . . . . . . . . . . . . . . . . . . . . . . . 13

10 Spatio-temporal Characteristics . . . . . . . . . . . . . . . . . . . . . . . . . . . . . . . . . 14

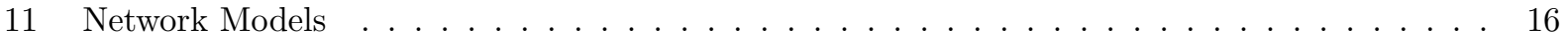

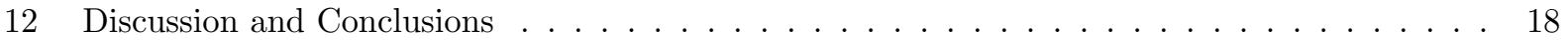

13 Acknowledgments . . . . . . . . . . . . . . . . . . . . . . . . . . . 19 


\section{Introduction}

Gun violence is a central public concern in the United States, annually leading to the deaths of 36,000 individuals and the non-fatal injuries of 85,000 others [28]. It has been called an epidemic and a public health crisis $[3,7,29]$. For infectious disease epidemics and associated public health planning (including recent Zika and Ebola outbreaks), officials relied on mathematical models to evaluate immediate responses and develop preventative policies $[29,39,67,70,73]$. There is also a long history of mathematical modeling in epidemiology that has provided a core body of underlying theory $[4,35]$. These theories are often used to develop responses and policies to mitigate epidemics. The construction of policies to curb the spread of gun violence could benefit from the development of mathematical models linked with available data.

In May of 2019, a diverse group of researchers participated in a workshop at the University of Tennessee, Knoxville. This workshop was sponsored by the Center for the Dynamics of Social Complexity (DySoC) and the National Institute for Mathematical and Biological Synthesis (NIMBioS). The objectives of this workshop were to (i) review the existing approaches on the mathematics and modeling of gun violence, (ii) identify and prioritize areas in the field that require further research, (iii) develop cross-disciplinary collaborations to gain new perspectives, and (iv) suggest research and data collection that could assist evidence-based policy recommendations. The focus was on these issues in the U.S..

The workshop incorporated discussions and critiques of the existing approaches to gun violence modeling and how these relate to the objectives for which models could be developed. Comparisons of various modeling approaches (including dynamical systems, agent-based, spatial, and statistical) and the parameterization of these models were considered. Through discussions of existing and future models, we also assessed the available data and suggested new data collection. The workshop considered the variety of scales at which models can be developed and the associated implications at these different scales. Relationships to models for human behavior, including those from social psychology and game theory, were considered.

Effective approaches to building a theory of gun violence, which will then inform a science of gun violence, requires perspectives from multiple disciplines. The workshop considered a systems approach that brought together interacting factors and components operating on multiple scales of time and space. Attendees incorporated ideas from various quantitative fields (including mathematics, computer science, statistics, and informatics), social science areas (including geography, economics, psychology, and criminology), and biological disciplines (including behavior, medicine, and ecology). A tenet was that necessary research should be informed by practitioners involved in policy and law enforcement and will account for ethical issues of social justice and privacy.

The purpose of this report is to present some of the responses to the mentioned objectives and to suggest areas of future research. Due to the wide variety of participant backgrounds, the identified future research areas are fairly broad and can be approached in different fields using different methods. Cross-disciplinary collaboration will improve the outcomes of research projects in this area.

NIMBioS was established in 2008 with an award from the National Science Foundation (NSF) and brings together researchers from around the world to collaborate across disciplinary boundaries to find creative solutions to today's complex biological problems. DySoC promotes transdisciplinary research into the origins, evolution, and futures of human social complexity through theory, data, and synthesis. NIMBioS is supported by the National Science Foundation through NSF Award \#DBI-1300426, with additional support from The University of Tennessee, Knoxville. Any opinions, findings, and conclusions or recommendations expressed in this material are those of the author(s) and do not necessarily reflect the views of the National Science Foundation.

\section{Motivation}

Approximately $60 \%$ of firearm deaths are suicides, with gun violence and gun crime contributing to the remaining $40 \%$ of gun deaths in the U.S. [21]. Apart from the obvious loss of human life, gun violence costs the United States $\$ 229$ billion each year [56]. Along with the physical trauma associated with fatal and non-fatal gun violence and gun crime, studies have shown that witnesses exposed to gun crime have 


\section{Key Influences in the Mathematics of Gun Violence}

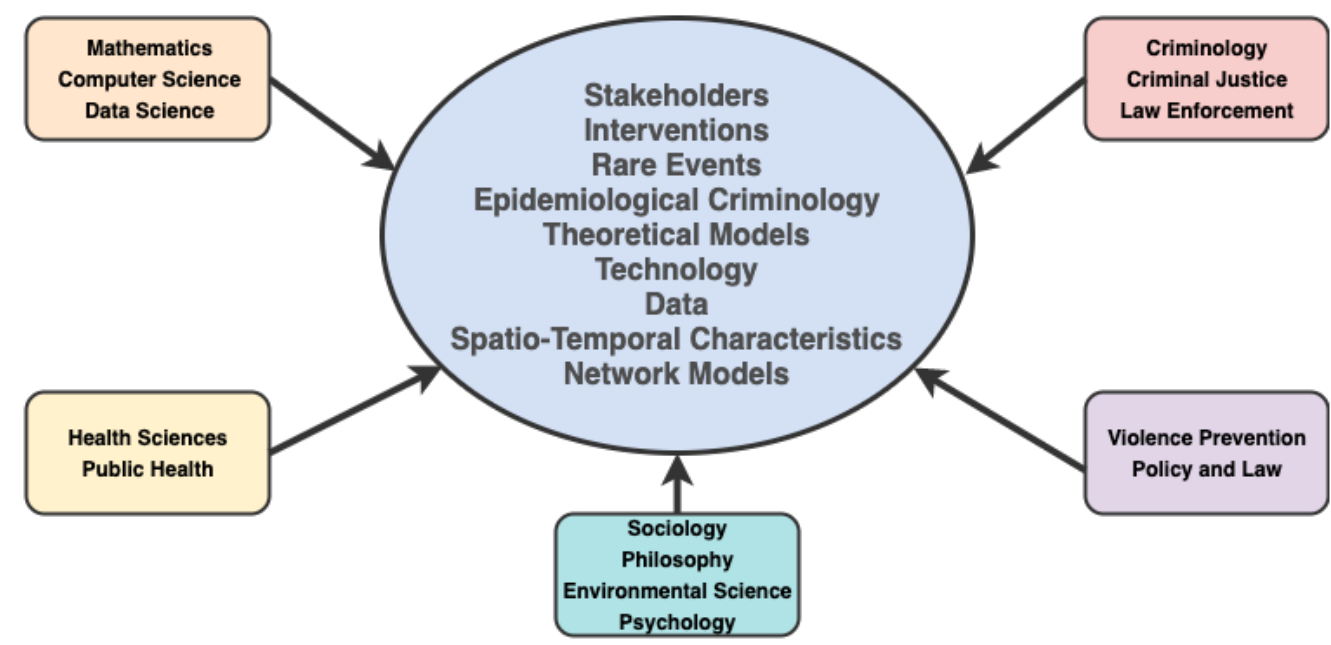

Figure 1: The key academic groups that contribute to the mathematics of gun violence.

increased risk of a number of health conditions including chronic obstructive lung disease (COPD), hepatitis, and stroke $[17,27]$. Youth exposed to community violence have significantly increased risk of engaging in dangerous behaviors such as ecstasy use, use of substances during sex, codeine use, and involvement in the juvenile justice system [69]. It is impossible to ignore that this is a major problem in the United States. These issues all contribute to the broad public concern with gun violence [9, 18, 22].

Despite the major effects that gun violence and gun crime have on individuals, research on the topic has received little federal funding and it is therefore severely under-studied. In 1993, Arthur Kellerman published a study finding that having a gun in the home was significantly associated with increased risk of homicide occurring in the home [36]. In response to this and other research studies on gun ownership and use, Congress passed the Dickey Amendment. This states that the CDC cannot use any federal funding to promote or advocate for gun control. Similar language was applied to the NIH funding appropriations in 2011. Although this amendment does not directly stop any research on gun violence or gun crime, it did reduce the amount of funding available for these projects. As a result, gun violence and gun crime research has received little support. Despite having similar mortality rates, gun violence research receives only $0.7 \%$ of the research funding of sepsis $[65,71]$.

In this report, we describe the topics that were covered during breakout sessions during the workshop. In each section we will review the relevant background information, present some of the major perspectives discussed during the session, and list the future directions within that topic to provide a starting point for future research projects. The breakout topics were chosen in two ways. Prior to the workshop, ideas were suggested by participants and co-organizers. These topics were then voted on to determine the top six options. At the workshop, further topics were suggested and chosen from, giving a total of nine topics. Each breakout group met twice with different participants to promote diversity of ideas. Google Docs were used to take notes on existing literature, commentary on the topic, and future questions in the field. 


\section{Workshop Participants}

This report would not have been possible without the expertise, contributions, and feedback from the workshop participants. The participants were:

- Tim Akers, Center for Research Innovation and Advocacy, Morgan State University

- Jeremy Auerbach, Environmental \& Radiological Health Sciences, Colorado State University

- Richard Block, Sociology, Loyola University Chicago

- Heather Brooks, Mathematics, University of California, Los Angeles

- Shani Buggs, Violence Prevention Research Program, University of California, Davis

- Cassandra Crifasi Johns Hopkins Center for Gun Policy and Research, Johns Hopkins University

- Grant Drawve, Sociology, University of Arkansas

- Stephen Eubank, Virginia Biocomplexity Institute \& Initiative, University of Virginia

- Nina Fefferman, Mathematics and Ecology \& Evolutionary Biology, NIMBioS and University of Tennessee, Knoxville

- Jason Goldstick, Emergency Medicine, University of Michigan; Director of Statistics \& Methods Section, Research Core, UM Injury Prevention Center

- Louis J. Gross, Mathematics and Ecology \& Evolutionary BIology, NIMBioS and University of Tennessee, Knoxville

- Natalie Hipple, Criminal Justice, Indiana University, Bloomington

- Jonathan Jay, FACTS Consortium, Public Health, Harvard University

- Charles Loeffler, Criminology, University of Pennsylvania

- Brianna Mills, Harborview Injury and Prevention Center, University of Washington

- George Mohler, Computer and Information Science, Indiana University, Purdue University Indianapolis

- Kellen Myers, Ecology \& Evolutionary Biology, University of Tennessee, Knoxville

- Veronica Pear, Violence Prevention Research Program, University of California, Davis

- Nancy Rodriguez, Applied Mathematics, University of Colorado, Boulder

- Jessica Saunders, Regulation, Evaluation, and Governance, Stanford University

- Shelby M. Scott, Ecology \& Evolutionary Biology, University of Tennessee, Knoxville

- Gary Slutkin, Public Health, University of Illinois at Chicago

- Rosanna Smart, Policy, RAND Corporation

- Christopher Strickland, Mathematics, University of Tennessee, Knoxville

- Jeffrey Taxman, Psychiatry, Medical College of Wisconsin

- Miriam Thalos, Philosophy, University of Tennessee, Knoxville

- Sherry Towers, Biosocial Complexity Initiative, Arizona State University

- Melissa Tracy, Epidemiology, Biostatistics, State University of New York, Albany

- Alexandria Volkening, NSF-Simons Center for Quantitative Biology, Northwestern University 


\section{Collaboration with Stakeholders}

\section{Summary}

Determining how stakeholders at all levels impact the perpetuation and interruption of crime and violence allows us to better create and implement policies to reduce the negative impacts of these events.

\section{Background}

There is not a broad literature on the relationship of stakeholders to gun crime and gun violence. Stakeholders can encompass any individuals or groups with a specific interest in this topic. This can include political figures, community members, health professionals, and a variety of others. Despite the fact that these groups are often concerned with the state of gun crime and gun violence, we know very little about their influence on the outcomes. The research for which stakeholder involvement has been most obvious is in intervention attempts.

Abt suggests a theoretical framework for preventing community violence among youth [1]. He posits that there is a particular need for stakeholders to be engaged when attempting to control community violence, specifically among youth [1]. Because stakeholders in these areas often know more about the system than policy makers, they can provide information about which interventions may or may not be effective for a given population [1]. The American Psychological Association (APA) produced a report with similar conclusions [6]. There is a need to include all types of stakeholders in preventative measures when it comes to gun violence specifically, both interpersonal and self-inflicted, to best-reduce the occurrence of these events [6]. Some research focuses on specific stakeholders and stakeholder groups. Sierra-Arevalo et al. evaluate Project Longevity, an intervention program in Chicago, IL, and discuss the impacts when law enforcement, social services, and community members are brought together to meet with members of violent street groups [62]. Involvement of stakeholders contributes to the reduction of group member involved incidents in New Haven, Connecticut, confirming the need for further exploration into focused deterrence interventions [62]. Grunwald and Papachristos observe an intervention in Chicago and find that bringing federal, state, and local law enforcement together with researchers and community organizations does help to reduce the amount of violence in a given area [31]. This study also identifies how the efficacy of interventions can change over

time, especially when the involved stakeholders are not consistent over the years [31]. This highlights the need to plan for personnel change for interventions to remain effective.

\section{Perspectives}

When dealing with stakeholders in issues of gun crime and gun violence, it is important to identify key players and how they interact with both the problem at hand and the community itself. Stakeholders can exist at any level, from local social groups to government entities, but the influence of stakeholders can shift and change over time. Analyzing the network of stakeholders involved in a community may improve the efficacy of interventions, and if the network structure can be generalized to other communities, it may make interventions more successfully transportable between systems. One of the major challenges in understanding this network concerns gathering appropriate data on stakeholder involvement and appreciation of various communication methods.

\section{Future Directions}

Several open questions regarding stakeholder collaborations were suggested. Some identify specific modeling techniques, while others are open ideas in need of further exploration with a number of methods.

- Are there common patterns in stakeholder networks that can be generalized across different communities? 
- Can we determine how changes in stakeholders affect intervention efforts and overall crime and violence within a community?

- In collaboration with stakeholders, are there methods of constrained optimization, such as optimal control theory that can feasibly be applied to show how a specific intervention minimizes costs, but maximizes reduction in crime and violence?

- Would a multi-scale model of stakeholders operating at a number of organizational and societal levels be appropriate to assess top-down and bottom-up interventions and interactions?

- Are compartmental models appropriate to track the interactions between different agents working towards a common goal and assist in maximizing the efficacy of these agencies in combating crime and violence?

\section{Intervention}

\section{Summary}

Crime and violence are complex societal problems with potentially complex interventions. Analyzing how different interventions affect different types of violence can allow for multi-faceted, evidence-based approaches to be implemented.

\section{Background}

Interventions are the most often considered and perhaps researched area in the gun violence and gun crime fields. Some researchers observe the efficacy of implemented interventions, others test the ability of theoretical interventions to adequately reduce violence, or suggest options without directly considering the outcomes. Gun violence and gun crime interventions are varied, but all share a similar goal - reduce the frequency and number of these events while simultaneously reducing the negative impacts of these programs (including costs, damaged relationships between law enforcement and community members, etc.). Some suggested and tested interventions are policy-based and involve legislation, while others suggest changes in society or communities.

Some studies consider the need for stakeholder involvement in interventions [1,6,31,62]. Generally, each of these highlights the need for community involvement when suggesting interventions. In terms of policybased interventions, Davis et al. list a number of policy-based interventions that should be taken into account, specifically by epidemiologists in the context of gun crime and gun violence [24]. These recommendations mainly focus on the need for organizations to collect data and produce research on firearm violence in the United States, and the need for epidemiologists to be involved in these studies to better inform interventions [24]. Cerdá et al. evaluate the effects of misdemeanor policing on homicide in New York City [19]. They find that misdemeanor policing does reduce homicide rates, but physical disorder in a given area is not a mediator of the impact of such policing [19]. Other forms of policing have also been evaluated. Saunders et al. explore the impacts of creating a Strategic Subjects List (SSL) to reduce gun violence in Chicago [57]. They find that subjects on the SSL are not significantly more or less likely to becomes a victim of violence, but are more likely to be arrested for a shooting [57]. Several laws have been passed in hopes of reducing gun crime and gun violence in a given area, including background checks and firearm prohibition laws. Morral et al. evaluate the strengths and weaknesses of different models for the effects of gun policies on outcomes [48]. This review categorizes gun policies and considers outcomes from these policies to find those which result in significant effects and the conclusions that can be drawn from a specific policy [48]. Similar reviews consider either specific policies or the impacts of these policies in specific areas [23, 58, 61].

Apart from policies, interventions have been used in hopes of reducing gun crime and gun violence. Some interventions involve focused deterrence, which seeks to change offender behavior by understanding underlying crime-producing dynamics and conditions that sustain recurring crime problems by implementing 
a blended strategy of law enforcement, community mobilization, and social service actions [12]. Braga et al. evaluate group-based and place-based methodologies in detecting direct and spillover crime reduction impacts of focused deterrence in Oakland, California [12]. They find that focused deterrence is a fairly good strategy for directly reducing crime and that there is also spillover into surrounding areas of this reduction [12]. Earlier studies observed the displacement of crime correlated with intervention. Guerette and Bowers examine 102 evaluations of situationally focused crime-prevention projects to determine the extent to which crime displacement was observed [32]. Displacement was defined as crime moving from one space to another after implementation of an intervention, whereas diffusion was defined as benefits spreading to a new location after implementation [32]. They found that an almost equal percentage of interventions resulted in both displacement and diffusion, but that often the displacement effects were mitigated by the treatment effects [32]. Brantingham et al., use mathematical modeling to quantify the magnitude of retaliation arising from gang crimes eunder different types of interventions and find that these violence interruption programs can significantly reduce gang retaliations [13]. Wiley et al. also use mathematical modeling, but from a more abstract perspective to assess some of the mechanisms that make violence interruption successful [70]. Using ordinary differential equations (ODEs), they find that targeting all potential violence transmitters can reduce gun violence more than targeting only gun-owning individuals, indicating that the benefits of focused deterrence depend on the groups targeted [70]. There are also a variety of hospital-based violence intervention programs that focus on intervening at the hospital and post-discharge level in order to break the cycle of violence [51]. These programs reduce health care costs by decreasing recidivism to the emergency room and also reduce societal costs [51].

Other research has assessed the impact of different policing methods on crime and violence reduction. Kondo et al. assess how greening lots in a city affects the crime levels in Youngstown, $\mathrm{OH}$ [37]. They find that "greening" abandoned lots by clearing debris and converting them into green spaces for the community helps to reduce crime and violence [37]. When these interventions are practiced, it is also important to evaluate the shifts in attitudes of community members, rather than solely observing the impact on crime [44]. Milam et al. evaluate the changes in attitudes that occurred a year after the Safe Streets intervention was implemented in Baltimore, Maryland [44]. They find that there were significant improvements in attitudes towards violence in the intervention community following implementation [44]. A number of studies observe the effects of community interventions, and consider how interventions on the individual level affect the propagation of violence. Taxman outlines a conceptual model that takes into account developmental, cultural, and social forces in concert with developmental neurophysiology to posit why specific individuals become violent [66]. These alternative forms, evaluations, and scales of intervention are creative means of solving a complex problem. Further innovation in developing interventions will lead to more effective policies and programs.

\section{Perspectives}

Interventions are a major study area in crime and violence. Before interventions are implemented, consideration should be given to how they will affect the system and how the effectiveness of an intervention will be evaluated. Generally, there is not much consensus as to how interventions are evaluated, both before and after implementation. There is also a major need to consider what types of violence a given intervention addresses. What is appropriate for self-inflicted harm may not be appropriate for mass shootings, so the focus for interventions is critical. Consideration of the root causes of crime and violence, and not strictly focus on reducing the outcome is necessary. Overall, there is a need for a multifaceted approach that can address both individual and population level intervention effects and outcomes.

\section{Future Directions}

Some of the many unexplored research questions for interventions include:

- Can we create a classification or taxonomy for interventions based in a taxonomy for different types of violence? 
- How do the interventions focus on the outcome of gun crime and violence, versus emphasizing upstream factors and root causes that could address gun crime and violence?

- Is there a consensus on how interventions are evaluated so that they might be compared across different systems?

\section{Quantifying the Impact of Rare Event Interventions}

\section{Summary}

In order to address rare events, we first need to define rarity. Then methods from other disciplines might be applied to analyze the occurrence of and intervene in rare event cases involving gun violence and gun crime.

\section{Background}

Few papers focus on gun violence and gun crime as rare events, but studies have related gun violence and gun crime to rare events, or analyze the more rare subsets of these crimes that occur. Despite the limited formal literature on this topic, the rarity of these events is an issue researchers in the field regularly face.

The American Psychological Association (APA) outlined how psychologists are integral to the prevention of gun violence and gun crime at a number of different levels [6]. The report focused on the fact that mental health care can help to reduce violence overall, but stresses that most individuals suffering from a mental illness are not violent [6]. Individual level interventions can prevent singular events from occurring [6]. When determining causes for mass shootings, mental illness is often presented, which may have been ameliorated but for the broader stigma surrounding mental health in the United States [43]. There is little populationlevel evidence that supports the notion that individuals diagnosed with mental illness are more likely than others to commit gun crimes [43]. In general, mass shootings and school shootings occur less frequently than interpersonal gun crimes and can be called "rare" in relation to other crimes, despite being far more publicized than other types. Towers et al. test for contagion behavior in mass shootings and school shootings and find that both show evidence of contagious behavior [67]. They report an increase in the probability of mass killings and school shootings for 13 days following an event [67]. Statistical analysis indicates that mass killings incite at least 0.30 new incidents, while school shootings incite at least 0.22 new incidents [67].

Viewing violence events as contagious makes it possible to borrow from other fields modeling rarity to determine similarities and differences. Mohler et al. investigate the extent to which an epidemic-type aftershock sequence (ETAS) forecasting model can outperform classic hotspot maps [45]. Since earthquakes are rare, ideas from geology msy similarly predict where future crimes might occur [45]. They find that ETAS models predict 1.4-2.2 times as much crime as other existing methods [45]. Improvised Explosive Devices (IEDs) are used with reasonable rarity in the Iraqi war [47]. Determining algorithms that can rank spatio-temporal event hotspots well for both IED events and for crime and traffic incidents can determine how these events are similar and how they differ [47]. The algorithm presented by Mohler is able to improve the percent of events captured in hotspots by up to $35 \%$ compared to other methods for crime and IED event data [47]. Using several rare events methods may assist in analyzing the dynamics observed and proposing better intervention methods.

\section{Perspectives}

Rarity is context dependent, both in terms of scale and field of study. Firearm homicides and earthquakes are rare within the criminology and geology fields, but if they both occurred as frequently as firearm homicides, geology would differ from current practice considerably. Rare events introduce uncertainty into datasets,

arising in two major types of rarity: large deviation processes and outliers. Mathematically, these types of rarity are analyzed using different methods. Analysis of rare events through aggregation methods to increase sample sizes can introduce errors. 


\section{Future Directions}

Some open questions in rare events research for gun violence and gun crime include:

- How transferable are the methods other disciplines use for rare events to gun violence and crime?

- What assumptions do various approaches for rare events make and how are they reasonable for firearm violence?

- What are some potentially better ways to identify and predict rare events before they occur in order to improve intervention?

\section{Epidemiological Criminology}

\section{Summary}

Analyzing crime and violence as epidemic processes requires considering the specific nuances and limitations of this analogy. Once circumstances for an apt analogy are determined, approaches from other fields may be appropriate.

\section{Background}

There is a formal paradigm known as "epidemiological criminology," which gracefully integrates the two disciplines [3]. This addresses a wide range of issues that affect the health and well-being of society [3]. It allows for theoretical model building, investigation practice and inquiry, and identification of unique populations and practical applications [3]. This intersection between health and public policy can assist different sub-disciplines. An example is the spread of incarceration through a social network [40]. Lum et al. present an agent-based model of incarceration based on the classic susceptible - infectious - susceptible (SIS) epidemic model [40]. They find that this model can replicate the racial disparities in incarceration rates if spread through a social network is included [40]. Other network models have been used in this field, some of which are mentioned in Section 11.

Aside from formal epidemiological criminology, other means of applying epidemiological and public health methods to gun violence and gun crime include those at the national level( e.g. Towers 2015 [67]). Policy development benefits from determining how epidemiology and public health can be involved in the prevention of firearm violence [24]. The International Network for Epidemiology in Policy (INEP) make a number of policy recommendations to reduce firearm violence [24]. These include making data more broadly available (even internationally), providing more funding for research, encouraging researchers to engage in multidisciplinary firearm prevention research, creating evidence-based policies, and encouraging public health practitioners to use epidemiological and other scientific evidence to create curricula [24]. It can also be helpful to apply epidemiological frameworks to create and evaluate interventions. Cure Violence is an intervention which uses a public health approach in order to change individual and community attitudes and norms about gun violence [16]. By considering gun violence as a communicable disease, the Cure Violence program attempts to stop the transmission of violent behavior in three ways: interrupting transmission directly, identifying and changing the thinking of potential transmitters, and changing group norms providing violence [16]. The Cure Violence program shows evidence of success at the level of jurisdictions or communities, but the results could not be disentangled from the national and regional trends in violent crime [16]. Violence reduction programs using public health frameworks, can be more effective than others [16]. Similar methods were applied Cali, Colombia, in the early 1990s [30]. By considering the associated risk factors, the mayor was able to reduce interpersonal conflict and determine other means of reducing gun crime in the city [30].

A major issue academics have with the analogy between gun violence and epidemics is defining the characteristics of gun violence and gun crime that allow it to fit the criteria of a disease. Slutkin presents the ways in which gun violence fits the basic infectious disease framework and how this framework can inform programs of violence reduction [63]. Three main characteristics of infectious diseases within a population are 
clustering, spread, and transmission [63]. Violence exhibits each of these characteristics [63]. Violence fits the criteria of a disease at the population level, and at the individual level, including: susceptibility, exposure, infectivity, transmission, incubation, latency, pathogenesis, inapparent/subclinical, carriers, a clinical spectrum, cure, and relapse [63]. Considering violence as an epidemic at both of these levels allows for more evidence-based interventions to be applied to areas most at risk or most affected by gun violence and gun crime [63].

\section{Perspectives}

The Epidemiological Criminology Framework is a specific set of strategies that has been used in Baltimore, Maryland [3]. The disease analogy for gun violence and crime requires care to ensure it is apt. Using epidemiology and disease ecology, including mathematical and statistical modeling, to analyze the characteristics of crime and violence may often be appropriate. Creating multi-scale models that connect the individual scale to population and community scales may be just as effective as it has been shown to be in disease ecology.

\section{Future Directions}

A group from the workshop is working on a commentary paper discussing the Epidemiological Criminology framework and intend to develop multi-scale models. Aside from this effort, other questions to be explored include:

- Is there a particular characteristic of gun violence that differentiates it from other forms of violence?

- Can we better understand the immunology surrounding gun violence and determine where the analogy to cancer or disease start and end? Which assumptions are appropriate and which are not?

\section{Theoretical Models}

\section{Summary}

There is no single model for all types of violence, but analyzing the factors and interactions that push an individual or group from non-violence to violence, even in the absence of data, can provide insight for crime and violence interruption. Theoretical models based on key concepts from a variety of disciplines can improve response to these events.

\section{Background}

There would be benefits from a cohesive theory or set of theories for gun violence and gun crime. Currently, there are theories from criminology, epidemiology, sociology, and other disciplines that are applied to crime, but few of them have a firm basis in both quantitative methods and criminology.

The Health Belief Model (HBM) contains several primary concepts that predict why people will take action to prevent, screen for, or control illness [20]. The concepts include susceptibility, seriousness, benefits and barriers to a behavior, cues to action, and self-efficacy [20]. In public health applications, this can be used to consider why a patient may not adhere to appropriate treatment regimens. From a crime perspective, this theory can be used to consider why an individual may carry a gun, perform a crime, and what their reaction to the consequences for their actions might be. Slutkin applies theories from epidemiology treating gun violence as a contagious disease [63]. One uses the Kermack-McKendrick theory, which predicts the number and distribution of cases of an infectious disease as it is transmitted through a population over time 
[64]. A basic ordinary differential equation (ODE) form of this is the SIR model represented by:

$$
\begin{aligned}
\frac{d S}{d t} & =-\frac{\beta I S}{N}, \\
\frac{d I}{d t} & =\frac{\beta I S}{N}-\gamma I, \\
\frac{d R}{d t} & =\gamma I,
\end{aligned}
$$

where $S$ is the number of susceptible individuals, $I$ is the number of infectious individuals, and $R$ is the number of removed or recovered individuals [64]. The transitions between states are due to the effective contact rate, $\beta$ (movement from susceptible to infectious), and the recovery rate, $\gamma$ (movement from infectious to removed) [64]. This method can be applied to, albeit with perhaps different mathematical forms, model the transitions of individuals in a population from non-violent to violent, similar to Wiley [70].

Theories from sociology and criminology are also used in the theoretical basis of crime. Within criminology, differential association theory was originally introduced in 1947 and proposes 8 hypotheses regarding crime as a learned behavior [15]. Bruinsma tested these propositions empirically to find that they can explain $51 \%$ of the variance in criminal behavior and that only three of the postulated relationships are rejected [15]. This theory related to the theory of planned behavior whereby beliefs are linked to behavior [2]. Planned behavior theory postulates that performing a behavior depends jointly on appraisal of a behavior (attitude), ability (behavioral control), and societal pressure (social norm) [2]. If all of these are appropriately met, then an individual will move towards intention (motivation) and then into the behavior itself [2]. According to this theory, all of these factors must be aligned in order to produce criminal behavior.

\section{Perspectives}

Each type of violence has different characteristics and these differences should be taken into consideration when creating mathematical and statistical models. These differences exist on a number of different scopes and scales, which affect what makes an individual likely to transition from non-violence to violence. The characteristics that may make someone more likely to commit violence can start in youth and, over time, a combination of social factors, environmental stressors, and other influences can lead to violent behavior. Ideas from sociology and psychology can help to determine the appropriate specific interactions within an individual, but accessing the specific datasets of these biomarkers and environmental conditions is difficult. While data availability is a major issue in gun violence and crime research, using general theories from a variety of disciplines can help to fill the gaps in the datasets by providing insight about generalizable concepts and their interactions which affect the transition to violence.

\section{Future Directions}

This session addressed what makes an individual perpetrate a violent act and how gun violence and crime can be treated as an epidemic (or whether it can). Some of the questions that still need to be addressed include:

- Can we generalize models for all types of crime and violence and how do we need to be discerning about the type of crime and violence being addressed?

- How do life stage characteristics of an individual, from childhood to adulthood and factors such as environmental stressors and social interactions, lead to violence in an individual, and how can we model these events and interactions?

- Can general theories from other disciplines be used to assist in developing insight about violent behavior even when data availability is greatly constrained?

- How can we best communicate the advantages and limitations of particular models and the methods used to evaluate them? 


\section{Technology}

\section{Summary}

When new technologies relevant to issues of gun violence are available and adopted, it is important to consider the specific purpose of the technology, how it will be evaluated for efficacy, and how its use will impact not only the social system as a whole, but also the individuals subject to the new technology.

\section{Background}

Technology is being increasingly used to observe, predict, respond and intervene in gun violence and gun crime. The technologies are in place, but the ethics surrounding data collection, analysis, and access are not yet defined. Renda and Zhang evaluate the use of an acoustic gunshot locator system (AGLS) to determine calls for service in Louisville, Kentucky [55]. They observe the differences between gunshots picked up through the AGLS and service calls made to 911 and find that there is underreporting of gun crime in the study area, enhancing the potential benefits of using AGLS in cities [55]. Loeffler and Flaxman use AGLS data to assess the spatio-temporal spread of gun crime (further discussion of these methods below) [39]. Ratcliffe et al. analyzes the ability of AGLS to aid in police incident reports [54]. They find that while gunshot incidents increased after the implementation of AGLS, there was no significant increase in the number of confirmed shootings [54]. Regarding crime prediction, several algorithms attempt to determine where crimes might occur in order to deploy resources. Mohler et al. demonstrate how predictive policing can help to reduce the amount of crime in Los Angeles, California [45]. While there are a number of technologies in place to assess gun violence and gun crime, not many analyses have been done to evaluate the effects of these technologies.

\section{Perspectives}

Technology deployment can impact gun violence and crime on several scales. This can include individual level technologies for gun access, community level technologies for policing, and national level technologies for more global analysis of gun crimes and violence. It is necessary to understand the specificity and sensitivity of these technologies and how they might feedback to the system in question. When introducing a new technology, it is important to determine its purpose. Is it being used for prevention, intervention, observation, direct response, or follow-up, and how does its purpose impact how it is tested and evaluated? It is also important to consider the policies and procedures that are related to the technology itself and the data collected. Ethical issues and bias concerns, especially when data is being used in order to predict individual activity or profile specific individuals, must be considered.

\section{Future Directions}

As access to sophisticated technologies increases, diverse research questions surrounding this technology arise including:

- Is there a taxonomy of the available technologies and their applications to different aspects of violence and crime?

- How are new technologies tested and evaluated for efficacy and utility? Is there a need to standardize this process?

- What can we learn from how various fields use technology to improve use of technology in the criminal justice framework?

- What could be done to create a meta-analysis of technology adoption and its efficacy in different communities?

- Can multi-scale modeling be used to explore the adoption of technology itself? 
- What policies and procedures must be in place in order to make sure technology is being used appropriately?

\section{Data Use}

\section{Summary}

There is a general lack of data availability in these systems, and the quality of the available data is questionable. In light of this, interdisciplinary collaborations are particularly important to fill gaps in data through theory and methods from other fields.

\section{Background}

Creation of evidence-informed policies and interventions for gun violence and gun crime would benefit from effective use of available data. Studies have looked at data use, treatment of data, and have made recommendations regarding better data collection. Regarding data use, Faust and Tita look at the issues that can occur when using social networks in criminological research [26]. They make recommendations and encourage researchers to use social network data and models in criminology, but to proceed with caution [26]. Associated with social networks are social media relationships that often underlie connections [52]. Social media data can provide a way to generate and access information about certain communities and then to determine how, when, and why gun violence might occur [52]. Data are highly important when attempting to develop interventions at the local level [42]. Using data to produce collaborative crime intelligence programs resulted in reductions in shootings between 2016 and 2017 in Chicago, illustrating the benefits of data-driven evidence-based policy creation [42].

Data use requires careful treatment of the data, from determining how to use the data ethically to dealing with imperfect and incomplete datasets. Because there are often identifiers within crime reports, the publicly available data can be poor. Nguyen et al. present a learning machine classifier that forecasts crime types when data are incomplete [50]. They find that by under-sampling the majority class of crimes to fix a problem of imbalance, the various predictive models tested perform better than with the original dataset [50]. This provides a way to fix issues in imbalanced data. Spatial analyses of crime also require special attention. Some spatial analyses tools use regression, but the characteristics of spatial data break the assumptions of most regressions [5]. Anselin et al. discuss concerns in using certain statistical tests for spatial data and how these practices can be rectified [5]. One of the major reasons for the poor quality of gun violence and gun crime data is the lack of a comprehensive research program in the United States [49]. In many ways, this could be due to the causality dilemma: the lack of programs prevents new data sources from arising, but the lack of data also prevents new programs from arising. Reducing gun violence and gun crime would benefit from appropriate and standardized data sources throughout the United States, to give researchers access to existing datasets, and to improve the datasets already in collection [49].

The available data are often collected without standardization or the collection techniques are not well documented. This can lead to poor data analysis and mis-interpreted results. Lauritsen and Cork discuss the need to improve crime data and crime statistics to provide more insight on crime reduction in the United States [38]. Specifically, the national crime statistics are not released in time to determine whether local crime patterns are part of broader regional or national patterns or whether increased crimes (especially homicide) are related to other characteristics of the local environment [38]. Public health officials also support improving data collection. Campos-Outcalt identifies the need to treat gun violence as a public health crisis, which notes that collecting data and maintaining a comprehensive database will help to improve the ways in which the United States deals with gun violence as a nation [18]. Improving data use, treatment, and collection can improve our capacity to analyze the causes of gun crime and gun violence, and potentially reduce these more effectively. 


\section{Perspectives}

Two major issues with data on crime and violence are data availability and data quality. Generally, we (as a society) do a good job at tracking births and deaths, but life cycle components between those are not as accurately measured. Some data sets are useful, but are difficult or nearly impossible to effectively collect. One example of this is measuring demographics at the level of interventions. As with other topics discussed in this report, there are trade offs between precision, realism, and generalizability in the data collected and the applications of these data sets. Specifically, there are issues when the data available are at a fine scale and the conclusions drawn are broad (atomistic fallacy) and also when the data available are at a broad scale, but the conclusions drawn are at a narrow scale (ecological fallacy) [41]. Some of the most important components of gun violence and gun crime are mostly absent from the available data and therefore previous work has used proxy measures for items such as gun ownership, the number of overall guns, and other elements of gun ownership and possession. Overall, collaboration of diverse scientists would to bring together content expertise and methodologies, especially when the content informs the model and there are limitations of what can and cannot be measured.

\section{Future Directions}

There is an urgent need for more data related to gun violence and crime. As with new technologies, it is important to think about the purpose of data collection and whether ethical considerations are taken into account when using this data. Some of the major open questions surrounding this topic are:

- Can we model the use of feedback data from interventions to improve their effectiveness after implementation, similar to the adaptive monitoring and management used for natural resources [33]?

- Is there a common framework that could be established for describing and collecting data from interventions for gun violence and gun crime? How do we design this to improve the ability to compare interventions?

- How do incomplete and poor quality datasets impact the types of models used and the assumptions made within these models, and the implications drawn from them? How might we design error and uncertainty analysis for gun crime and violence models?

- There is a need for the following datasets at various temporal and spatial scales:

- Fine-scale demographic data

- Intervention implementation, evaluation, and outcomes

- Gun ownership

- Number of guns

- Non-fatal firearm injuries

- Details on weapons used to perpetrate a crime

\section{Spatio-temporal Characteristics}

\section{Summary}

Incorporating spatial, temporal, and spatio-temporal data for violence and crime analysis requires approaches that consider scales of aggregation and accounts for differing data quality across scales. 


\section{Background}

Many studies of gun violence and gun crime have focused on the spatio-temporal distribution of events. Based on the purpose of the study, these can be grouped into: defining hotspots in a given area, assessing intervention attempts, understanding the places associated with crime, and creating methods for spatiotemporal analysis. Hotspots are often used to understand where crime density is high and hotspot analysis can be used to distribute resources and apply interventions. Short et al. create a reaction-diffusion model for all types of crime and determine how hotspots displace and dissipate in a geographic area [60]. They find that there are types of hotspots that can be displaced and others that can be mitigated with interventions and therefore will not spread [60]. Shiode et al. observe the patterns of crime types in space, time, and spacetime dimensions to analyze the variation of crime incidents [59]. They then determine how different types of hotspots can persist in a given area and how these results can be used to better target police interventions [59]. Different methods are used to determine the significance and dynamics of hotspots. Mohler uses a marked point process model to create more accurate hotspot maps of crime in Chicago, Illinois [46]. By adding in leading indicator crime types, both long- and short-terms crime patterns can be better captured [46]. Loeffler and Flaxman use a similar methodology to determine whether gun violence in Washington, D.C. shows contagious behavior in space, time and spatio-temporal dimensions [39]. The Bayesian spatiotemporal point process is applied to acoustic gunshot locator system (AGLS) data and minimal contagion is found for this dataset [39]. The model has the form:

$$
\lambda(x, y, t)=m_{0} \mu(x, y, t)+\theta \sum_{i: t_{i}<t} \omega \exp \left(-\omega\left(t-t_{i}\right)\right) \frac{1}{2 \pi \sigma^{2}} \exp \left(-\left(\left(x-x_{i}\right)^{2}\right)+\left(y-y_{i}\right)^{2} /\left(2 \sigma^{2}\right)\right),
$$

where $\lambda$ is the conditional intensity of gun crime in both space $(x, y)$ and time $(t), m_{0}$ is a weighting component, $\mu(x, y, t)$ is a background intensity, $\theta$ is the proportion of crimes incited by past crimes, and $\sigma$ and $\omega$ are length scale parameters for space and time, respectively. This model includes a Bayesian component whereby some of the parameters have prior distributions placed on them to better approximate the dynamics of the model [39].

Once hotspots of crime are located, this information can be used to create targeted interventions that may reduce crime. Cerdá et al. determine how misdemeanor policing reduces crime using a spatio-analytic test [19]. They find that misdemeanor policing reduces homicide rates, but there is no evidence for physical disorder associating with homicide rates [19]. Brantingham et al. test the effects of violence interruption on retaliatory gang crimes [13]. Using multivariate spatio-temporal point process models, they find that intervention methods cut retaliatory gang retaliations by $45.3 \%$ [13]. Another community intervention involves taking vacant lots and "greening" them by removing debris and putting down grass in order to create community spaces [37]. After adjusting for sociodemographic factors, treating lots resulted in a significant reduction in all crime types [37]. Thus some studies illustrate how the use of spatio-temporal information to plan interventions can help optimize resource use to reduce crime.

Research on connections between places and crime have existed for decades. Brantingham and Brantingham defined crime attractors and generators in the mid-1990s, and further studies have elaborated on this work to determine landmarks that may show increased crime density [14]. One such study looked at the effects of crime generators, crime attractors, and offender anchor points on the distribution of street robberies in Chicago [10]. Bernasco and Block find that blocks hosting crime attractors and generators not only have elevated numbers of robberies themselves but also tend to radiate their elevated crime risk to adjacent blocks [10]. This supports the spatial contagion claims often made in the literature. Phan et al. look at the relationship between certain landmarks and the spatial and temporal distribution of street crime [53]. They mainly observe the adult entertainment area of Boston and find that even though the area itself has declined during the study period, street crimes did not show significant decline [53]. Also in Boston, Braga et al. consider the stability of crime at "micro-places," represented by street segments and intersections [11]. They find that the majority of crime hotspots at this scale are fairly stable, but that it may be helpful to study the $3 \%$ of hotspots that are volatile to determine the dynamics of spread and propagation [11]. Schnell et al. add support to this idea by replicating a study done in The Hague, The Netherlands [58]. Applying 
these methods to data from Chicago, Illinois, shows that the majority of the variability in crime incidents can be attributed to street segments [58]. All of these studies focus on the scale at which crime should be studied and the ways in which specific businesses and landmarks may influence crime in a given area.

Analysis of concentration and scales of crime are important, but there is also a need for new tools to further assess crime and violence distributions. Diverse methods exist for analyzing hotspots, but there is no consensus on the appropriate technique for different research questions. Drawve et al. compares the accuracy and precision of risk terrain modeling (RTM) to the accuracy and precision of nearest neighbor hierarchical (Nnh) methods, as they are applied to a dataset from Little Rock, Arkansas [25]. They find that while RTM is more precise than Nnh, Nnh is more accurate than RTM [25]. This illustrates the need to consider the purpose of a test is before committing to a method. As mentioned in Section 5, Mohler introduces CrimeRank, which maximizes the amount of crime captured by the top ranked hotspots, specifically used for complex geometries [47]. Such techniques allow for analysis of the spatial distribution of crime and potentially impact strategies for response.

\section{Perspectives}

Many quantitative models created for crime and violence consider spatio-temporal aspects of these events, but several issues should be taken into account when creating and assessing these models. One of these is scale. Due to data quality and quantity issues, it is often difficult to match the scales of spatial, temporal, and spatio-temporal data. It is important to be intentional about model choice and the conclusions drawn. There is also an understandable desire to generalize results from one region or time period to another. This highlights the need to be clear about the purpose, assumptions, and limitations of each model. With spatial, temporal, and spatio-temporal data, measurement error should be assessed when creating models. Determining the impact of measurement error on model conclusions and actionable recommendations is important.

\section{Future Directions}

Spatio-temporal modeling of violence and crime requires caution due to the variety of different issues mentioned above. Some further issues to consider are:

- Is there a classification for city types dependent upon scales that allows us to better assess and intervene for crime and violence?

- How does the movement of individuals within a city affect how crime occurs and propagates throughout that city and how can it be modeled?

- Can we develop modeling approaches that estimate and characterize the generalizability across scales and diverse regions?

- How do we account for both systematic and random measurement error at various scales in our models?

- What affect do differing scales of data (spatial, temporal, and spatio-temporal) have on the models we create and how do we take these differences into account?

\section{Network Models}

\section{Summary}

Determining how networks on different scales impact the perpetuation and interruption of crime and violence can improve how violent and criminal events are assessed and how they may be reduced. Networks consider the connections between different nodes within a common framework and various metrics then can characterize the properties of the collective. 


\section{Background}

While the media tends to focus on mass shootings and school shootings, the majority of gun violence and gun crime (that is not self-inflicted) is interpersonal, so network connections influence the propagation of crime. Gun violence and gun crime cannot occur without access to a firearm, so gun spread throughout social networks is critical. Hureau and Braga analyze the spread of guns through a high-risk network to determine how the types of guns and the prices of guns differ when obtained illegally [34]. They find that guns in high-risk networks are often more expensive and are older than those obtained legally [34]. Despite the poor quality of the existing gun ownership data, studies such as these help to uncover the dynamics of illegal guns within networks.

Focused deterrence, mentioned in Section 3 and further discussed in Section 4, requires identifying gang ties and disrupting violence from spreading between these nodes. Braga et al. analyze how focused deterrence strategies influence gun violence in Oakland, California [12]. They find that network-informed intervention methods reduce shootings in treated groups when compared to untreated groups [12]. They also evaluate the spillover of deterrence effects and find that the intervention spills over into other areas with varying magnitudes [12]. Therefore, there is not only a spread of violence in space and time, there is also a spread of non-violence. Wood and Papachristos elaborate on this idea, focusing on the direct and spillover effects of an intervention program in Chicago, Illinois [72]. Leveraging network connections leads to a $3.2 \%$ reduction in victimization through direct effects, while the potential spillover effects reduce victimization $1.5 \%$ [72]. Leveraging network structure in the context of intervention can produce greater reduction in gun violence than unstructured interventions [72].

Though each network may have idiosyncrasies, analyzing a variety of networks allows researchers to explore commonalities and identify differences that may cause interventions and reduction policies to affect certain networks in different ways. Bastomski et al. analyze the role of neighborhood-level criminal networks in shaping the distribution of crime throughout cities [8]. Considering the networks at the neighborhood-level, particularly with co-offenders, indicates that a neighborhood's embeddedness increases the local homicide rate [8]. Another Chicago study uses epidemic models to determine whether social networks can be used to explain and predict gun violence [29]. The probabilistic contagion model indicates that gunshot violence follows an epidemic-like process of social contagion that is transmitted through social networks [29]. Violence prevention efforts that account for contagion and demographics have the potential to prevent more shootings than efforts that focus solely on demographics [29]. These studies are absent from the review by Tracy et al. due to publication dates [68]. Their review examines weapon-related violence in a variety of networks and finds that exposure to a victim or perpetrator of violence in one's networks is correlated with one's own probability of being a gunshot victim or perpetrator [68].

\section{Perspectives}

Discussions about network models of violence and crime have mainly focused on networks of individuals and how these lead to criminal and violent events. Some of the networks of concern include outreach networks, social networks, and other community networks. There are features within these networks including the culture and social norms surrounding gun ownership, storage, and use - that may increase the risk of an individual choosing to perpetuate a criminal or violent act towards others or themselves. Determining the structure of these networks and how they are linked to events can help to intervene in these situations. An issue in creating network models is identifying the feature sets and obtaining data on the specific characteristics that relate to an individual's propensity to commit a violent or criminal act and their vulnerability to be a victim of these acts. There is also a need to consider how social media impacts the discussions surrounding violence and crime and how this might be added to mathematical and statistical models.

\section{Future Directions}

Some potential questions that might be addressed with network methods include: 
- Can we identify individual and group characteristics of secondary violence networks?

- How can we evaluate the efficacy of outreach networks in the context of intervention?

- Is there a network of gun availability that can assist in modeling how guns are obtained and then used in violent and criminal acts?

- Can we identify the role of information transmission through social media on the propagation and prevention of gun violence? More generally, what is the effect of social media on public opinion about gun violence?

\section{Discussion and Conclusions}

This workshop considered many components of gun violence. The objectives were to (i) review the existing approaches on the mathematics and modeling of gun violence, (ii) identify and prioritize areas in the field that require further research, (iii) develop cross-disciplinary collaborations to gain new perspectives, and (iv) suggest research and data-collection that could assist evidence-based policy recommendations. The first objective was addressed through the webinar preceding the workshop. The webinar is available at: http://www.nimbios.org/workshops/WS_gunviolence. A review paper of quantitative models addressing gun violence and gun crime will be submitted for publication in the coming months. The second objective was addressed by extensive breakout session discussions at the workshop, leading to the sections of this report. New collaborations have begun to form. The workshop did not make formal recommendations for research and data-collection, but this report gives a starting point for many further projects.

There were some common threads woven in each of the breakout sessions. One of these is the need for more cross-disciplinary and inter-disciplinary work. This includes collaborations between researchers from different fields, drawing from a wide variety of disciplines to achieve a better grasp on what is occurring in the dynamics of gun violence and gun crime. For each topic discussed above, references arose from diverse fields. Gun violence research draws from sociology, epidemiology, philosophy, geography, and seismology to better discover causes and solutions. Further unexplored connections will no doubt lead to new approaches and insights. Leveraging diverse expertise across a wide range of fields can completely alter the ways in which quantitative methodology is used to address these major issues in public health and law enforcement.

Both mathematical and statistical models of gun crime and gun violence have been created, but there are limitations to these models and there are many types of models that could be applied to these systems. Cellular automata models are discrete in both space and time, two characteristics that can also define gun violence and gun crime, but these models have rarely if ever been used in this context. Difference equation models may assist in dynamic analysis and in quantitative criminology contexts. Better metrics of evaluation and comparison of models to determine how well they represent the system. There are some ways to compare models and understand what best represents the system, but there is no consensus within the field as to the best way to carry this out. Evaluating the models is absolutely important, but it is equally important to have metrics for evaluating and comparing intervention techniques for gun crime and gun violence.

A consensus from the workshop participants is that data improvements are imperative for advancing the field. There is a lack of overall data available, especially on gun ownership and the details surrounding what causes an individual to move from non-violent into a violent space. The data that are available are often poorly documented and described, which can impact the interpretation of results. Caution should be taken when using available datasets and there needs to be better documentation about how the data are collected, cleaned, and processed. The limitations of datasets should be addressed in research, which uses them to make policy and intervention decisions.

All of these issues matter for research on gun violence and gun crime, but none of this research is possible without funding. If there is a desire to reduce the frequency of gun crime and gun violence events using evidence-based policies, funding needs to be provided to the appropriate groups. Government groups should be given the monetary support and ability to take part in gun violence research, with other groups given funding to support their work. This can include non-profit groups, industry teams, and academic research 
centers. Providing intentional and directed grants specifically for gun violence research will help improve a number of the issues discussed throughout this workshop and, hopefully, contribute to the reduction of gun violence events.

\section{Acknowledgments}

The author would like to thank Louis J. Gross for his comments and guidance when writing this report, the workshop participants for their contributions, and NIMBioS for hosting the workshop. The author acknowledges support from a National Defense Science and Engineering Graduate Fellowship. NIMBioS is supported by the National Science Foundation through award DBI 1300426 to the University of Tennessee with further support from the University of Tennessee. Any opinions, findings, and conclusions or recommendations expressed in this material are those of the author(s) and do not necessarily reflect the views of the National Science Foundation. 


\section{Bibliography}

[1] Thomas P. Abt. Towards a framework for preventing community violence among youth. Psychology, Health $\&$ Medicine, 22:266-285, 2017. PMID: 27973915.

[2] Icek Ajzen. The theory of planned behavior. Organizational behavior and human decision processes, 50(2):179-211, 1991.

[3] Timothy A. Akers and Mark M. Lanier. "epidemiological criminology": Coming full circle. American Journal of Public Health, 99(3):397-402, 2009. PMID: 19150901.

[4] R. Anderson and R. May. Population biology of infectious diseases: Part 1. 280:361 - 367, 1979.

[5] Luc Anselin, Jacqueline Cohen, David Cook, Wilpen Gorr, and George Tita. Spatial Analyses of Crime, volume 4, pages 213-262. 2000.

[6] American Psychological Association. Gun violence: Prediction, prevention, and policy. Technical report, Americal Psychological Association, 2013.

[7] Gia Barboza-Salerno. A secondary spatial analysis of gun violence near boston schools: a public health approach. Journal of Urban Health, 95(3):344-360, 042018.

[8] Sara Bastomski, Noli Brazil, and Andrew V. Papachristos. Neighborhood co-offending networks, structural embeddedness, and violent crime in chicago. Social Networks, 51:23-39, 2017.

[9] Pamela Behrman, Colleen A. Redding, Sheela Raja, Tamara Newton, Nisha Beharie, and Destiny Printz. Society of behavioral medicine ( $\mathrm{sbm}$ ) position statement: restore cdc funding for firearms and gun violence prevention research. 8(6):958-961, 2018.

[10] Wim Bernasco and Richard Block. Robberies in chicago: A block-level analysis of the influence of crime generators, crime attractors, and offender anchor points. Journal of Research in Crime and Delinquency, 48(1):33-57, 2011.

[11] Anthony A. Braga, Andrew V. Papachristos, and David M. Hureau. The concentration and stability of gun violence at micro places in boston, 1980-2008. Journal of Quantitative Criminology, 26(1):33-53, 2010.

[12] Anthony A. Braga, Greg Zimmerman, Lisa Barao, Chelsea Farrell, Rod K. Brunson, and Andrew V. Papachristos. Street gangs, gun violence, and focused deterrence: Comparing place-based and groupbased evaluation methods to estimate direct and spillover deterrent effects. Journal of Research in Crime and Delinquency, pages 1-39, 2019.

[13] P. Jeffrey Brantingham, Nick Sundback, Frederick Paik Schoenberg, Andrea L. Bertozzi, Joshua Gordon, Jorja Leap, Krinstine Chan, Molly Kraus, Sean Malinowski, and Denise Herz. Does violence interruption work? PNAS, 8(7), 2018.

[14] Patricia Brantingham and Paul Brantingham. Criminality of place: Crime generators and crime attractors. European Journal on Criminal Policy and Research, 13:5-26, 1995. 
[15] G.J.N. Bruinsma. Differential association theory reconsidered: An extension and its empirical test. Journal of Quantitative Criminology, 8(29), 1992.

[16] Jeffrey A. Butts, Caterina Gouvis Roman, Lindsay Bostwick, and Jeremy R. Porter. Cure violence: A public health model to reduce gun violence. Annual Review of Public Health, 36(1):39-53, 2015. PMID: 25581151.

[17] T. Rashad Byrdsong, Angela Devan, and Hide Yamatani. A ground-up model for gun violence reduction: A community-based public health approach. Journal of Evidence-Informed Social Work, 13(1):76-86, 2016. PMID: 26151769.

[18] Doug Campos-Outcalt. Treat gun violence like the public health crisis it is. The Journal of Family Practice, 66(11):653, November 2017.

[19] Magdalena Cerdá, Melissa Tracy, Steven F. Messner, David Vlahov, Kenneth Tardiff, and Sandro Galea. Misdemeanor policing, physical disorder, and gun-related homicide: A spatial analytic test of "brokenwindows" theory. Epidemiology, 20(4):533-541, 2009.

[20] V.L. Champion and C.S. Skinner. The Health Belief Model, pages 45-65. Jossey-Bass, 2008.

[21] Joint Economic Committee. A state-by-state examination of the economic costs of gun violence, 09 2019.

[22] Karen S. Cox. A public health crisis: recommendations to reduce gun violence in america. 66(3):219-220, 2018.

[23] Cassandra K. Crifasi, Alexander McCourt, and Daniel W Webster. Policies to reduce gun violence in illinois: Research, policy analysis, and recommendations, 2019. Johns Hopkins Bloomberg School of Public Health Report.

[24] Amy B. Davis, James A. Gaudino, Colin L. Soskolne, and Wael K. Al-Delaimy. The role of epidemiology in firearm violence prevention: a policy brief. International Journal of Epidemiology, pages 1-5, April 2018 .

[25] Grant Drawve, Stacy C. Moak, and Emily R. Berthelot. Predictability of gun crimes: a comparison of hot spot and risk terrain modelling techniques. Policing and Society, 26(3):312-331, 2016.

[26] Katherine Faust and George E. Tita. Social networks and crime: Pitfalls and promises for advancing the field. Annual Review of Criminology, 2(1):99-122, 2019.

[27] Vincent J. Felitti, Robert F. Anda, Dale Nordenberg, David F. Williamson, Alison M. Spitz, Valerie Edwards, Mary P. Koss, and James S. Marks. Relationship of childhood abuse and household dysfunction to many of the leading causes of death in adults: The adverse childhood experiences (ace) study. American Journal of Preventive Medicine, 14(4):245 - 258, 1998.

[28] Centers for Disease Control. Web-based injury statistics query and reporting system (wisqars). Online, 2017.

[29] Ben Green, Thibaut Horel, and Andrew V. Papachristos. Modeling contagion through social networks to explain and predict gunshot violence in chicago, 2006 to 2014. JAMA Internal Medicine, 2017.

[30] Alyssa Greenhouse. The science of violence prevention. Bulletin of the World Health Organization, 93(10):665-740, October 2015.

[31] Ben Grunwald and Andrew V. Papachristos. Project safe neighborhoods in chicago: Looking back a decade later. Journal of Criminal Law and Criminology, 107:131-160, 012017. 
[32] Rob T. Guerette and Kate J. Bowers. Assessing the extent of crime displacement and diffusion of benefits: A review of situational crime prevention evaluations. Criminology, 47(4):1331-1368, 2009.

[33] C.S. Holling. Adaptive Environmental Assessment and Management. John Wiley \& Sons, 1978.

[34] David M. Hureau and Anthony A. Braga. The trade in tools: The market for illicit guns in high-risk networks. Criminology, 56(3):510-545, 2018.

[35] Matt J. Keeling and Pejman Rohani. Modeling Infectious Diseases in Humans and Animals. Princeton University Press, 2008.

[36] Arthur L. Kellermann, Frederick P. Rivara, Norman B. Rushforth, Joyce G. Banton, Donald T. Reay, Jerry T. Francisco, Ana B. Locci, Janice Prodzinski, Bela B. Hackman, and Grant Somes. Gun ownership as a risk factor for homicide in the home. New England Journal of Medicine, 329(15):1084-1091, 1993. PMID: 8371731.

[37] Michelle Kondo, Bernadette Hohl, SeungHoon Han, and Charles Branas. Effects of greening and community reuse of vacant lots on crime. Urban Studies, 53(15):3279-3295, 2016. PMID: 28529389.

[38] Janet L. Lauritsen and Daniel L. Cork. Modernizing Crime Statistics: Report 2: New Systems for Measuring Crime. The National Academies Press, Washington, DC, 2018.

[39] C. Loeffler and S. Flaxman. Is gun violence contagious? a spatiotemporal test. Journal of Quantitative Criminology, August 2018.

[40] Kristian Lum, Samarth Swarup, Stephen Eubank, and James Hawdon. The contagious nature of imprisonment: an agent-based model to explain racial disparities in incarceration rates. Journal of The Royal Society Interface, 11(98), 2014.

[41] JP Mackenbach. Roaming through methodology. xxvi. the ecological fallacy and its less well-known counterpart, the atomistic fallacy. Nederlands tijdschrift voor geneeskunde, 144(44):2097—2100, October 2000.

[42] Sean W. Malinowski and Marjolijn Bruggeling. Building an effective, data-driven, violent crime fighting program at the local level: Chicago's strategic decision support center model. DOJ Journal of Federal Law and Practice, 2018.

[43] Jonathan M. Metzal and Kenneth T MacLeish. Mental illness, mass shootings, and the politics of american firearms. American Journal of Public Health, 105(2):240-249, 2015.

[44] Adam J. Milam, Shani A. Buggs, C. Debra M. Furr-Holden, Philip J. Leaf, Catherine P. Bradshaw, and Daniel Webster. Changes in attitudes toward guns and shootings following implementation of the baltimore safe streets intervention. Journal of Urban Health, 93(4):609-626, Aug 2016.

[45] G. O. Mohler, M. B. Short, Sean Malinowski, Mark Johnson, G. E. Tita, Andrea L. Bertozzi, and P. J. Brantingham. Randomized controlled field trials of predictive policing. Journal of the American Statistical Association, 110(512):1399-1411, 2015.

[46] George Mohler. Marked point process hotspot maps for homicide and gun crime prediction in chicago. International Journal of Forecasting, 30:491-497, 2014.

[47] George Mohler, Michael D. Porter, Jeremy Carter, and Gary LaFree. Learning to rank spatio-temporal event hotspots. In Proceedings of the 7th international workshop on urban computing (URBCOMP2018), 2018.

[48] Andrew R. Morral. The science of gun policy: A critical synthesis of research evidence on the effects of gun policies in the united states, 2018. RAND Corporation Report. 
[49] Andrew R. Morral. Reducing disagreements on gun policy through scientific research and an improved data infrastructure, 2019. RAND Corporation Report.

[50] Trung T. Nguyen, Amartya Hatua, and Andrew H. Sung. Building a learning machine classifier with inadequate data for crime prediction. Journal of Advances in Information Technology, 8(2):141-147, 2017.

[51] National Network of Hospital-based Violence Intervention Programs. Hospital-based violence intervention: Practices and policies to end the cycle of violence. online, 2019.

[52] Desmond Upton Patton, Kyle McGregor, and Gary Slutkin. Youth gun violence prevention in a digital age. Pediatrics, 2018.

[53] Linh Phan, Nina Fefferman, Dora Hui, and Doug Brugge. Impact of street crime on boston chinatown. Local Environment, 15(5):481-491, 2010.

[54] Jerry H. Ratcliffe, Matthew Lattanzio, George Kikuchi, and Kevin Thomas. A partially randomized field experiment on the effect of an acoustic gunshot detection system on police incident reports. Journal of Experimental Criminology, 15, 2018.

[55] William Renda and Charlie H. Zhang. Comparative analysis of firearm discharge recorded by gunshot detection technology and calls for service in louisville, kentucky. ISPRS International Journal of GeoInformation, 8(6), 2019.

[56] Adrian Rivera. A call to action: Policy proposals to ameliorate gun violence in the us. The Trinity Papers (2011 - present), 2019.

[57] Jessica Saunders, Priscillia Hunt, and John S. Hollywood. Predictions put into practice: a quasiexperimental evaluation of chicago's predictive policing pilot. Journal of Experimental Criminology, 12(3):347-371, Sep 2016.

[58] Cory Schnell, Anthony A. Braga, and Eric L. Piza. The influence of community areas, neighborhood clusters, and street segments on the spatial variability of violent crime in chicago. Journal of Quantitative Criminology, 33(3):469-496, Sep 2017.

[59] Shino Shiode, Narushige Shiode, Richard Block, and Carolyn R. Block. Space-time characteristics of micro-scale crime occurrences: an application of a network-based space-time search window technique for crime incidents in chicago. International Journal of Geographical Information Science, 29(5):697$719,2015$.

[60] Martin B. Short, P. Jeffrey Brantingham, Andrea L. Bertozzi, and George E. Tita. Dissipation and displacement of hotspots in reaction-diffusion models of crime. Proceedings of the National Academy of Sciences, 107(9):3961-3965, 2010.

[61] Michael Siegel and Claire Boine. What are the most effective policies in reducing gun homicides?, 2019. Rockefeller Institute of Government Policy Brief.

[62] Michael Sierra-Arevalo, Yanick Charette, and Andrew V. Papachristos. Evaluating the effect of project longevity on group-involved shootings and homicides in new haven, connecticut. Crime 85 Delinquency, 63(4):446-467, 2017.

[63] Gary Slutkin. Violence is a contagious disease: Contagion of violence: Workshop summary, 2013.

[64] Robert Smith? Modelling Disease Ecology with Mathematics, volume 2 of AIMS Series on Differential Equations 83 Dynamical Systems. American Institute of Mathematical Sciences, 1 edition, 2008.

[65] D.E. Stark and N.H. Shah. Funding and publication of research on gun violence and other leading causes of death. JAMA, 317(1):84-86, 2017. 
[66] Jeffrey Taxman. Gun violence in america - a tri-vector model. International Journal of Applied Psychoanalytic Studies, 13(2):113-123, 2016.

[67] S. Towers, A. Gomez-Lievano, M. Khan, A. Mubayi, and C. Castillo-Chavez. Contagion in mass killings and school schootings. PLoS ONE, 10(7):117-259, 2015.

[68] Melissa Tracy, Anthony A. Braga, and Andrew V. Papachristos. The transmission of gun and other weapon-involved violence within social networks. Epidemiologic Reviews, 38(1):70-86, 2016.

[69] Dexter R. Voisin, Sadiq Patel, Jun Sung Hong, Lois Takahashi, and Noni Gaylord-Harden. Behavioral health correlates of exposure to community violence among african-american adolescents in chicago. Children and Youth Services Review, 69:97-105, 2016.

[70] Shari A. Wiley, Michael Z. Levy, and Charles C. Branas. The Impact of Violence Interruption on the Diffusion of Violence: A Mathematical Modeling Approach, pages 225-249. Springer International Publishing, Cham, 2016.

[71] Margaret A Winker, Kamran Abbasi, and Frederick P Rivara. Unsafe and understudied: the us gun problem. BMJ, 352, 2016.

[72] George Wood and Andrew V. Papachristos. Reducing gunshot victimization in high-risk social networks through direct and spillover effects. Nature Human Behavior, 2019.

[73] Jie $\mathrm{Xu}$ and Elizabeth Griffiths. Shooting on the street: Measuring the spatial influence of physical features on gun violence in a bounded street network. Journal of Quantitative Criminology, 33(2):237253, Jun 2017. 